\title{
A Rare Manifestation of Common Disease: Cardiac Cirrhosis
}

\author{
Richmond R Gomes* \\ Department of Medicine, Ad-din Women's Medical College Hospital, Bangladesh
}

*Corresponding author: Richmond Ronald Gomes, Department of Medicine, Addin Women's Medical College Hospital, Dhaka, Bangladesh.

Received Date: July 30, 2021

Published Date: August 26, 2021

\begin{abstract}
The relation between diseased heart and liver may manifests as acute liver injury, chronic congestive hepatopathy, even cardiac cirrhosis. Congestive hepatopathy caused from impaired blood return to the right ventricle with increased filling pressure. Chronic liver disease (CLD) is the most frequent presentation of hepatobiliary disease. Very rare cause, like long term right heart failure may also be a cause of underlying disease for CLD. We present a case of a 54-year-old female with cardiac cirrhosis. Initial workup was negative. Later thoracic imaging and echocardiography showed chronic obstructive pulmonary disease (COPD) with evidence of pulmonary hypertension. We will briefly discuss the literature on cardiac causes of liver cirrhosis. Our case will present such a short report or cardio-hepatic relations.
\end{abstract}

Keywords: Congestive hepatopathy; Cardiac Cirrhosis; CLD; COPD; Pulmonary hypertension

\section{Introduction}

Chronic right sided congestive heart failure may cause chronic liver injury and cirrhosis of liver but is very uncommon. In long term right heart failure there is elevated venous pressure that is transmitted to liver sinusoids via inferior vena cava and hepatic veins. This leads to long term passive congestion and relative ischemia due to poor circulation eventually leading to necrosis and fibrosis of liver predominantly of centrilobular region. Patient generally presents with clinical features of congestive heart failure and portal hypertension but very rarely presents with variceal hemorrhage or encephalopathy [1]. But our case patient presented with evidence of variceal hemorrhage. Also, the overall prognosis of cardiac cirrhosis is not well established, and treatment of cardiac cirrhosis is mainly aimed at managing underlying heart failure, so it becomes important to distinguish it from other cause of cirrhosis1. The timely diagnosis of a cardiac etiology of liver dysfunction is important because such dysfunction is potentially reversible if the underlying cardiac disease is treated before the development of frank cirrhosis [2,3].

\section{Case Report}

A 54 year old lady, home maker, hailing from rural Bangladesh, not known to have diabetes, hypertension or coronary artery disease, chronic smoker (beedi-local hand-rolled cigarettes) presented with progressively increasing abdominal distension for last 6 months, bilateral leg swelling for 1 month and H/O two episodes of passage of black tarry stool since then. On repeated enquiry she also revealed of chronic cough and breathlessness with winter exacerbation for last 10 years and episodes of pedal edema relieving after local medicine. There was no history of alcohol intake, high risk sexual behavior, jaundice, tuberculosis, long term drug or herbal intake, surgery, or blood transfusion. There was no significant family history. On general examination patient was cooperative and well oriented with poor nutrition. 
Pallor, mild icterus, and bi-pedal pitting edema was present. Cyanosis, clubbing, lymphadenopathy were absent. Pulse-70/ min regular, microvolumes, normal in character and vessel wall normal. Blood pressure-100/ 70 mmHg. Neck vein was engorged, and pulsatile and jugular venous pressure raised. On abdominal examination, abdomen was distended diffusely with eversion of umbilicus and prominent veins in flanks and epigastrium with blood flow from below upwards. Abdominal striae were seen. There were no scar marks. No superficial tenderness present. Splenomegaly was present of $4 \mathrm{~cm}$, firm, non-tender with smooth surface. Liver was also palpable $2 \mathrm{~cm}$ from right costal margin along right mid clavicular line, firm, tender with smooth surface and regular margin. No other lump present. Fluid thrill was present. On cardiovascular examination precordium seemed to be normal. Apex beat in 5 th intercostal space $2 \mathrm{~cm}$. Lateral to mid clavicular line normal in character. Thrill or para-sternal heave absent. On auscultation 1st and 2nd heart sound audible with loud pulmonary component of 2nd heart sound. The holosystolic, high-pitched, blowing murmur of tricuspid insufficiency best heard at the lower left sternal border. The murmur intensifies with inspiration and decreases with expiration. On respiratory examination chest bilaterally symmetrical with decreased movement on both sides. Trachea central and no deformity of spine seen. Respiratory rate of $26 / \mathrm{min}$. With use of accessory muscles seen. Vocal fremitus equal on both sides. Hyper-Resonant note heard on percussion. Bilaterally decreased breath sounds with diffuse rhonchi heard over lung fields. Vocal resonance decreased bilaterally. Nervous system examination reveals no abnormality.
Lab reports revealed: Heamoglobin-9.1 gm/dl, Total leucocyte count-3,800/dl, Differential count-neutrophil-58\%, Lymphocytes-30\%, Platelet's count-117,000/dl. Random Blood sugar-6.6 mmol/L, Serum Sodium-131 mmol/l, Serum Potassium-4.1 mmol/l, Serum Creatinine- $0.9 \mathrm{mg} / \mathrm{dl}$ Liver Function Test-S. Bilirubin-3.9 gm/dl, SALP-106 IU, SGPT-111 IU, Serum Protein-5.5 gm/dl, Serum Albumin-2.7 gm /dl Ascitic Fluid Examination-TLC-110/ cc, DLC-N35\% L65\%, Protein-1.3 gm/ dl, SAAG-1.4 ADA 12.1U/L (normal <30U/L) Prothrombin time: patient 18 secs, control 12 secs. Viral markers (HbsAg, HCV)Negative Chest X-ray-Cardiac Enlargement with accentuation of bronchovesicular marking bilateral mild pleural effusion, ECG-Rate of 70/min with regular rhythm, ABG-pH-7.38, pCO2-65, p02-74, Sp02-88\%, USG Abdomen-Liver-17.16 cm, coarse parenchyma, Portal vein-12.9 mm \& tortuous, Gross spleenomegaly-15.1 cm., Splenic vein-14.2 mm, tortuous \& dilated with multiple collaterals in perihilar splenic region. Gross peritoneal collection. 2-D EchoGrade 3 Tricuspid regurgitation, Severe Pulmonary Arterial Hypertension (PASP $40 \mathrm{~mm} \mathrm{Hg}$, dilated right ventricle and right atrium (Figure 1). Upper GI Endoscopy-Esophagus shows grade II $\times$ III columns of esophageal varices (Figure 2). Pro BNP was $15656 \mathrm{pg} / \mathrm{ml}$ (normal <400 pg/ml). TSH was normal $(0.829 \mathrm{IU} / \mathrm{ml}$, normal 0.350-3.40 IU/ml) Pulmonary Function Test-FEV1-52\%, FVC-79\%, FEV1/FVC-0.66 and improvement in FEV1 after use of bronchodilator was 7\% suggesting of chronic obstructive airway disease stage II of GOLD criteria. Fibroscan of liver; Median stiffness was $37.4 \mathrm{Kpa}$, IQR/MED-10\%, which correlate with stage-4 fibrosis, that is cirrhosis. Our final diagnosis was cardiac cirrhosis.

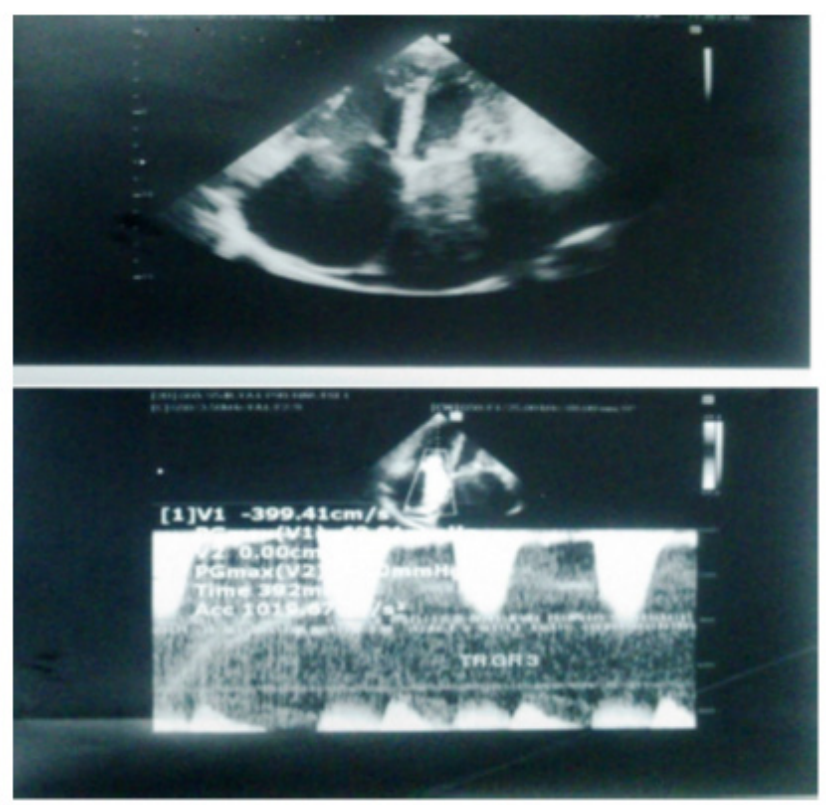

Figure 1: 2-D Echo-Grade 3 Tricuspid regurgitation, Severe Pulmonary Arterial Hypertension (PASP 40 mm Hg, dilated right ventricle and right atrium). 


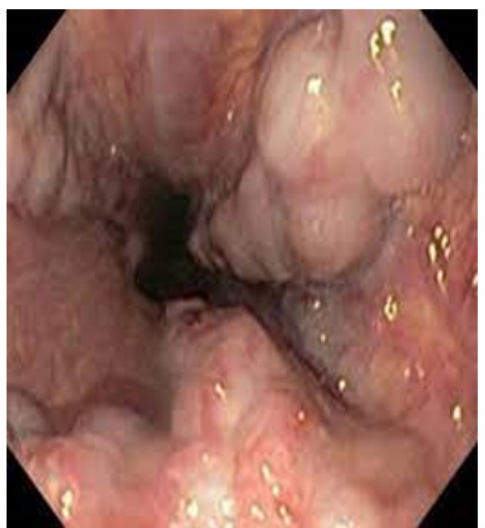

Figure 1: Upper GI Endoscopy-Esophagus shows grade II × III columns of esophageal varices respectively.

She was started salt and fluid restrictions (daily $1000 \mathrm{ml} /$ day) along with oral diuretics containing frusemide and spironolactone combination. Oral nitrates were advised to prevent further variceal bleeding as b-blockers are avoided in patients with respiratory airway diseases. Long acting b-agonist inhalers, montelukast and doxophyline were given to relieve broncho-constriction. Proton pump inhibitor prescribed to reduce acid production and prevent further damage due to acid reflux. Lactulose prescribed to prevent constipation and related complications. She was also transfused with 1 unit packed red cells and 4 units of fresh frozen plasma. Patient was given education regarding diet, precautions and follow up after discharge.

\section{Discussion}

Term cardiac cirrhosis denotes any type of hepatic fibrosis occurring in cardiac patient [4]. Our case report is in agreement with the previous observations of chronic liver injury due to long term congestive heart failure. It is a very uncommon cause of CLD and it's difficult to distinguish from other causes of liver cirrhosis [5]. The most important mechanisms responsible for the development of congestive hepatopathy are hepatic congestion, decreased hepatic blood flow and hypoxemia 5 followed by atrophy, necrosis of hepatocytes, thrombi resulting due to cholestasis [6]. Causes of cardiac cirrhosis are valvular heart disease, cardiomyopathy, pericardial disease, ischemic heart disease, primary lung disease [7]. With decrease in incidence valvular heart disease, cardiomyopathy in etiology of cardiac cirrhosis has increased [8].

Our case had primary lung disease due to chronic smoking which resulted in pulmonary hypertension leading to chronic congestive heart failure. This further leads to passive congestion and relative ischemia due to poor circulation eventually leading to necrosis and fibrosis of liver predominantly of centrilobular region [9]. Usually, cases of cardiac cirrhosis do not develop variceal hemorrhage or encephalopathy, but our case had unusual presentation of melena suggesting variceal bleeding. Our case had Obstructive airway disease of stage II according to GOLD10 staging evidenced from deranged Pulmonary Function Test, Abnormal Blood Gas analysis. Evidence of Pulmonary hypertension was evident clinically in form of loud P2 and murmur of tricuspid regurgitation which was established on 2D Echocardiography. Chronic congestive heart failure established on long history of 5 years for which he is taking treatment (? diuretics) from quack of which records were not available and raised pro-BNP level.

Later he developed congestive hepatopathy and signs of portal hypertension as evidenced by splenomegaly, progressive ascites which was transudative with SAAG> 1.113, jaundice, dyspnea, engorged neck vein, hepatomegaly, pedal oedema, normal alkaline phosphatase levels, raised AST, ALT and serum bilirubin. Metabolic and synthetic functions of liver were also compromised evident from decreased serum albumin and deranged PT/INR [7]. In congestive hepatopathy, liver function tests do not show the specific pattern as in patient with hypoxic hepatopathy [11]. Cholestatic enzymes together with low albumin and high bilirubin are the strongest risk factor for poor outcome, in case of chronic heart failure [12]. Chest X-ray was suggestive of congestive cardiac failure as there was bilateral pleural effusion. Splenomegaly was associated with hypersplenism as evident from pancytopenia in blood picture. As our patient was suffering from chronic congestive heart failure and ascites, transabdominal liver biopsy is at risk and Transjugular liver biopsy is not practiced at our setting for the evaluation of cirrhosis. So, fibro scan was done, and result was suggesting liver cirrhosis. Usually, cases of cardiac cirrhosis does not develop variceal bleeding, but our case presented with variceal bleeding evident from history of melana which was established on upper gastro-intestinal endoscopy in which therapeutic banding could not be done due to financial constraints [13].

The timely diagnosis of a cardiac etiology of liver dysfunction is important because such dysfunction is potentially reversible if the underlying cardiac disease is treated before the development 
of frank cirrhosis [2,3]. Moreover, early treatment of underlying cardiac disease might also prevent the development of hepatocellular carcinoma as suggested by an interesting case study in which a patient with negative hepatitis serologies and cirrhosis secondary to constrictive pericarditis developed hepatocellular carcinoma confirmed by biopsy [14].

\section{Conclusion}

This case study illustrates to gastroenterologists the need to consider a cardiac etiology in the work-up of cirrhosis especially when the most common causes are not found. A patient with COPD developing chronic right sided heart failure due to pulmonary hypertension causes passive congestion on hepatic veins, eventually lead to hepatic fibrosis, and raised portal hypertension. Though variceal bleed is uncommon in portal hypertension due to cardiac cirrhosis but may be presenting complain in rare case as seen in our case. Thought COPD and cardiac cirrhosis both are very uncommon, our interest was to highlight the cardiac cause should be evaluated in a dysphonic adult, where the causes of CLD were not certain.

\section{Acknowledgement}

None

\section{Conflict of Interest}

No conflict of interest.

\section{References}

1. Bacon BR (2012) irrhosis and Its Complications. In: Longo DL, Kasper DL, Jameson JL (eds.), Harrison's Principles of Internal Medicine $\left(18^{\text {th }}\right.$ edn), NewYork: Mc Graw Hill, 2(308): 2596-2597.

2. F Heureux, L Frankart, B Marchandise, M Buche, JP Martinet, et al. (1997) Recurrent ascites: two case reports. Acta Clinica Belgica 52(3): 176-181.
3. AA Sheth, JK Lim (2008) Liver disease from asymptomatic constrictive pericarditis. J Clin Gastroenterol 42(8): 956-958.

4. Blumgart HL, Katzin H (1938) Cardiac cirrhosis of the liver a clinical and pathological study. Trans Am Clin Climatol Assoc 54: 82-86.

5. Moller S, Hendrickson JH (2008) Cardiovascular complications of cirrhosis. Gut 57: 268-278.

6. Dai DF, Swanson PE, Krieger EV, Liou IW, Carithers RL, et al. (2014) Congestive hepatic Fibrosis score: A novel histological assessment of clinical severity. Mod Pathol 27: 1552-1558.

7. Kubo SH, Walter BA, John DH, M Clark, RJ Cody (1987) Liver function abnormalities in chronic heart failure. Influence of systemic hemodynamic. Arch Intern Med 147: 12227-12230.

8. Sekiyama T, Nagano T, Aramaki T (1994) Congestive (cardiac) cirrhosis. Nippon Rinsho 52(1): 229-233.

9. Sekiyama T, Nagano T, Aramaki T (1981) A clinic pathologic study of 1000 subjects at autopsy. Am J Pathol Aug 104(2): 159-166.

10. Pauwels RA, Buist AS, Calverley PM, C R Jenkins, SS Hurd, et al. (2001) Global strategy for the diagnosis, management, and prevention of chronic obstructive pulmonary disease. NHL $\neg$ BI/WHO Global Initiative for Chronic Obstructive Lung Disease (GOLD) Workshop summary. Am J Respir Crit Care Med 163(5): 1256-1276.

11. Mollaer S (2013) Bernardi Interactions of the heart and the liver. Eur Heart J 34: 2804-2811.

12. Chokshi AL, Cheema FH, Schaefle KJ, Jiang J, Collado E, et al. (2012) Hepatic dysfunction and survival after orthopic heart transplantation; application of the MELD scoring system for outcome prediction. J Heart lung Transplant 32: 591-600.

13. Runyon BA (1988) Cardiac arcites: A Ccharacterization. J Clin Gastroenterol 10: 410-412.

14. Pil Sang Song, Kwang Cheol Koh, Byung Chul Yoo, Seung Woon Paik, Joon Hyoek Lee, et al. (2005) A case of hepatocellular carcinoma complicating cardiac cirrhosis caused by constrictive pericarditis. Korean J Gastroenterol 45(6): 436-440. 